\title{
Mixed Infections of Plant Viruses in Crops: Solo vs. Group Game ${ }^{\dagger}$
}

\author{
Inmaculada Ferriol, Ornela Chase, María Luisa Domingo-Calap and Juan José López-Moya * \\ Centre for Research in Agricultural Genomics (CRAG, CSIC-IRTA-UAB-UB), 08193 Cerdanyola del Vallès, \\ Spain; inmaculada.ferriol@cragenomica.es (I.F.); ornela.chase@cragenomica.es (O.C.); \\ marialuisa.domingo.calap@gmail.com (M.L.D.-C.) \\ * Correspondence: juanjose.lopez@cragenomica.es \\ † Presented at Viruses 2020-Novel Concepts in Virology, 5-7 February 2020.
}

Published: 23 June 2020

\begin{abstract}
Plant diseases are responsible for considerable economic losses in agriculture worldwide. Recent surveys and metagenomics approaches reveal a higher than expected incidence of complex diseases, like those caused by mixed viral infections. Particularly, frequent cases of mixed infections are co-infections or superinfections of plant viruses belonging to different genera in the families Potyviridae (Ipomovirus or Potyvirus) and Closteroviridae (Crinivirus). The outcome of such multiple infections could modify viral traits, such as host range, titer, tissue and cell tropisms, and even vector preference and transmission rates. Therefore, we believe that understanding the virus-virus, virus-host, and virus-vector interactions would be crucial for developing effective control measures. Since there is still limited knowledge about the molecular mechanisms underlying the different interactions, and how they might contribute to specific diseases in mixed infection, we are analyzing ipomovirus-crinivirus and potyvirus-crinivirus pathosystems, to better understand single and mixed infections in selected susceptible hosts (Cucurbitaceae and Convolvulaceae plants), also incorporating in the study the interactions with insect vectors (whiteflies and aphids). Among other strategies, we are engineering new biotechnological tools, to explore the molecular biology and transmission mechanisms of several viruses implicated in complex diseases, and we are also addressing the possibility to produce virus-like particles (VLPs) through transient expression of the $\mathrm{CP}$ of different viruses in Nicotiana benthamiana plants, with the aim to study requirements for virion formation and determinants of transmission. Work supported by project AGL2016-75529-R and grant "Severo-Ochoa" SEV-2015-0533.
\end{abstract}

Keywords: ipomovirus; crinivirus; potyvirus; mixed infections; VLPs 